\title{
Use of CFD Technology in Hydraulics System Design for off-Highway Equipment and Applications
}

\author{
Shivayogi S. Salutagi, Milind S. Kulkarni, and Aniruddha Kulkarni
}

\begin{abstract}
Hydraulics technology is most preferred for off-highway equipment due to its high power density. Pumps, motors, valves, cylinders and hoses constitute a typical hydraulic machinery. Design of these parts will decide efficiency, reliability and power density of the system and the entire equipment itself. To meet these requirements, there is a paradigm shift towards the use of computational fluid dynamics (CFD) technology to design these products. CFD is used to simulate actual behavior of the fluid system, identify the critical areas of concern, evaluate various design alternatives and develop an optimized solution. The paper highlights CFD modeling approach with simulation challenges for pumps, motors and valves to predict pressure losses, fill speed and cavitation. Validation of CFD results with test data for key performance parameters is highlighted. This simulation methodology not only saves design and prototype building cost but also more importantly accelerates design cycle and improves speed to market. Thus, it has emerged as an integrated part of the design process.
\end{abstract}

Index Terms-Cavitation, CFD, fill speed, multiphase, motors, pressure drop, pumps, valves.

\section{INTRODUCTION}

A hydraulic system for off-highway comprises three major elements: hydraulic power generator such as pumps; transmitters and controllers such as hoses and control valves; and actuators such as motors and cylinders. The successful operation of any hydraulic system requires desired performance of all three elements. Therefore, it is important to understand the flow behavior in order to design the components and hence the system. Current industrial trend is towards the use of simulation technology (CFD) for detail investigation of flow physics and performance prediction. In this paper, three case studies are presented where the use of simulation is made to predict the performance. In first case, CFD simulation of a piston pump is elaborated for fill-speed and cavitation predictions. In second case, a hydraulic control valve is described for pressure override prediction. Performance prediction of a Geroler ${ }^{\mathrm{TM}}$ motor is discussed in third case.

\section{CASE STUdy - AXIAL Piston PUMP}

In hydraulic systems, axial piston pumps are used to deliver variable flow rate at required pressure. Fig. 1 shows typical piston pump architecture. Fill speed and cavitation are two

Manuscript received May 12, 2015; revised July 16, 2015.

The authors are with the Eaton Technologies Pvt. Ltd., Pune, 411014, India (e-mail: shivayogissalutagi@eaton.com, milindskulkarni@eaton.com, aniruddhakulkarni@eaton.com). important performance characteristics for piston pump. Fill speed is the measure of filling capability of suction chambers of the pump which determines maximum speed rating of the pump. It is the critical speed after which flow rate starts deviating from linearity with increase in speed, as shown in Fig. 2 [1]. If pump operates beyond its fill speed, cavitation will occur which will degrade the performance and reduces life of the pump. Fig. 1 shows the general flow passage of the piston pump and typical cavitation damage on valve plate. Prediction of fill speed and cavitation requires simulation of multiphase flow and cavitation physics along with modeling of complex pump dynamics. Multiphase modeling with pump dynamics poses challenges in handling numerical instabilities and convergence difficulties. This section explains modeling methodology regarding the simulation of piston pump.

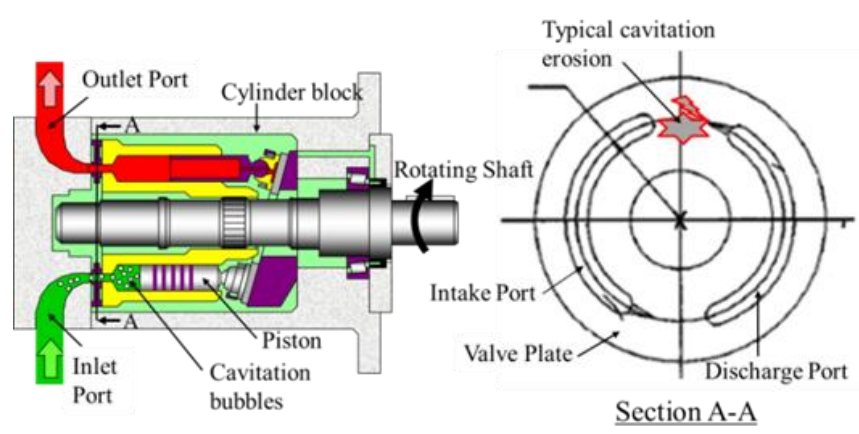

Fig. 1. Typical piston pump flow passage.

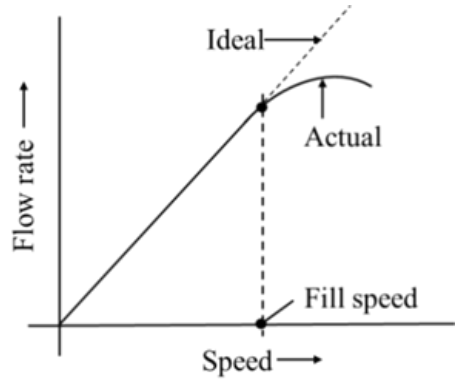

Fig. 2. Fill speed characteristics [1].

\section{A. Mathematical Background}

A two-phase cavitation model [2] is used to model the flow features along with a conventional $k-\varepsilon$ turbulence model. The liquid-vapor mass transfer is governed by the vapor transport equation:

$$
\frac{\partial}{\partial t}\left(\alpha \rho_{v}\right)+\nabla \cdot\left(\alpha \rho_{v} \vec{V}_{v}\right)=R_{e}-R_{c}
$$

where, $v$ is the vapor phase, $\alpha$ is the vapor volume fraction, $\rho_{v}$ 
is vapor density, $V_{v}$ is the vapor phase velocity and, $R_{e}$ and $R_{c}$ are mass transfer source terms connected to the growth and collapse of the vapor bubbles respectively. The net phase change rate or mass transfer rate uses the following two-phase continuity equations.

$$
\begin{gathered}
\text { Liquid phase: } \frac{\partial}{\partial t}\left[(1-\alpha) \rho_{l}\right]+\nabla \cdot\left[(1-\alpha) \rho_{l} \vec{V}\right]=-R \\
\text { Vapor phase: } \frac{\partial}{\partial t}\left(\alpha \rho_{v}\right)+\nabla \cdot\left(\alpha \rho_{v} \vec{V}\right)=R \\
\text { Mixture: } \frac{\partial}{\partial t}(\rho)+\nabla \cdot(\rho \vec{V})=0
\end{gathered}
$$

where, $l$ is liquid phase, where, $\rho_{l}$ is liquid density, $\rho$ is mixture density, and $R$ is the net mass source term is given as:

$$
R=\frac{\rho_{v} \rho_{l}}{\rho} \frac{D \alpha}{D t}
$$

Mixture density $(\rho)$ is defined as:

$$
\rho=\alpha \rho_{v}+(1-\alpha) \rho_{l}
$$

Combining equations (3), (4) and (5) yields a relationship between the mixture density and vapor volume fraction:

$$
\frac{D \rho}{D t}=-\left(\rho_{l}-\rho_{v}\right) \frac{D \alpha}{D t}
$$

The vapor volume fraction can be co-related from bubble number density and radius of bubble:

$$
\alpha=\frac{n_{b} \frac{4}{3} \pi R_{B}^{3}}{1+n_{b} \frac{4}{3} \pi R_{B}{ }^{3}}
$$

From above equations, net mass transfer rate $(R)$ is derived which is given below:

$$
R=\frac{\rho_{v} \rho_{l}}{\rho} \alpha(1-\alpha) \frac{3}{R_{B}} \sqrt{\frac{2}{3} \frac{\left(P_{v}-P\right)}{\rho_{l}}}, R_{B}=\left(\frac{\alpha}{1-\alpha} \frac{3}{4 \pi} \frac{1}{n}\right)^{\frac{1}{3}}
$$

where, $R_{B}$ is bubble radius, $P_{v}$ is vapor pressure, and $P$ is local pressure. The final form of above equation for modeling evaporation and condensation process is as follows:

When $P \leq P_{v}$, there will be an evaporation (i.e. vapor generation) which is modelled as:

$$
R_{e}=\frac{\rho_{v} \rho_{l}}{\rho} \alpha(1-\alpha) \frac{3}{R_{B}} \sqrt{\frac{2}{3} \frac{\left(P_{v}-P\right)}{\rho_{l}}}
$$

When $P \geq P_{v}$, there will be condensation (i.e. vapor collapse) which is modelled as:

$$
R_{c}=\frac{\rho_{v} \rho_{l}}{\rho} \alpha(1-\alpha) \frac{3}{R_{B}} \sqrt{\frac{2}{3} \frac{\left(P-P_{v}\right)}{\rho_{l}}}
$$

\section{B. CFD Flow Domain and Mesh}

Typical flow domain consists of suction and delivery kidney ports, valve plate and all nine piston chambers as shown in Fig. 3. Hexahedral mesh is created separately for each stationary and rotating components using ICEM CFD 15.0. Interface technique is used to connect multiple zones. Snap shots of a typical mesh of different parts are shown in Fig. 4. Total mesh count is $\sim 10$ lakh elements.
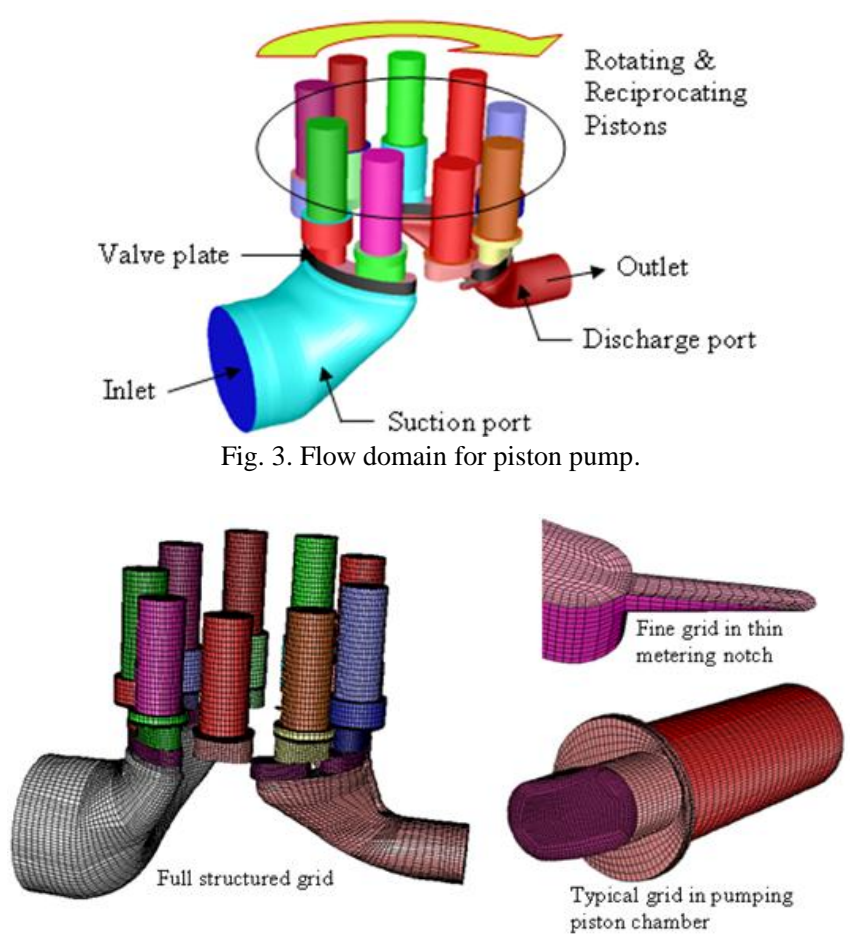

Fig. 4. Grid in the flow domain.

\section{CFD Modeling Approach}

\section{1) Modeling piston pump dynamics}

The cross sectional view of a typical piston pump [3] is shown in Fig. 5. Standard layering technique of dynamic deforming mesh is used to model the intake and delivery strokes of all nine pistons through user defined function (UDF) using the below equation.

$$
\text { Piston displacement, } x=r \times \tan (\alpha) \times \sin \left(\theta_{n}\right)
$$

where, $r$ is the piston pitch radius, $\alpha$ is the swash-plate angle and $\theta_{n}$ is the angular position of $n^{\text {th }}$ piston.

\section{2) Boundary conditions and fluid properties}

The pressure boundary condition is applied at inlet and outlet. Angular speed is defined at all piston zones for rotational motion. Fluid properties (density, viscosity, bulk modulus, and vapor pressure) of mineral oil are used for the simulations.

\section{3) Numerical models}

Cavitation model with two-phase flow is used with k- $\varepsilon$ turbulence model. Schnerr-Sauer model [4] is used to simulate cavitation phenomena for predicting filling capability of suctions chambers of pump. Further advances in the field of cavitation energy release model [5] are in progress. 


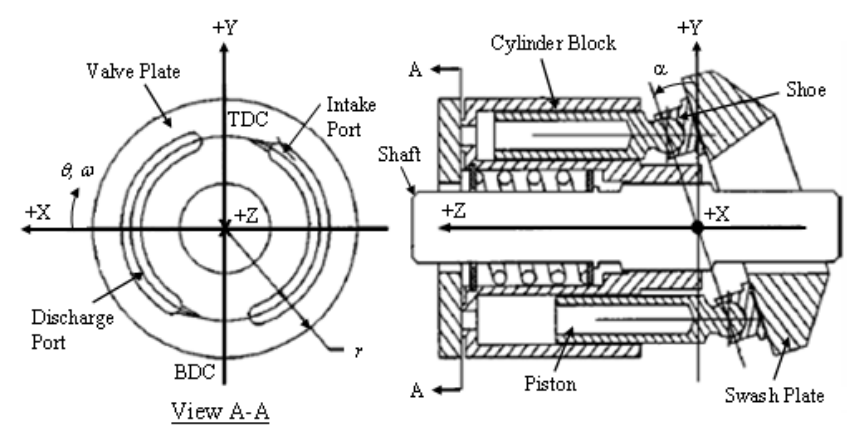

Fig. 5. Typical cross sectional view of axial piston pump.

\section{Results}

In this section, fill speed results are described. Two pumps of different designs are analyzed and the flow rate at different speeds is predicted. Comparison of test and CFD results for one of the pumps is shown in Fig. 6. It is observed that the flow rate starts deviating from linearity at $\sim 0.9$ normalized speed. This is due to initiation of cavitation i.e. formation of cavitation bubbles reduces the flow rate. Analysis predictions are in good agreement with test data with less than $6 \%$ error.

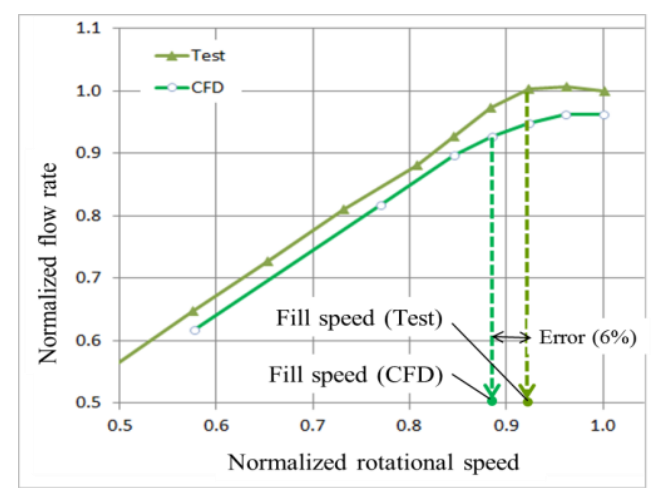

Fig. 6. Comparison of test and CFD fill speed characteristics.

The developed CFD approach is being applied for predicting fill speed and cavitation performance for different designs. Critical design factors such as valve plate timing angles, notch design, cross porting, cylinder barrel fluid cavity and kidney widths can be modified to improve the performance. Thus, the methodology is useful to study various design concepts which reduces physical testing of intermediate designs. This helps in saving significant amount of physical testing time and cost.

\section{CASE Study - Hydraulic Control Valve}

In this section, a poppet type relief valve performance prediction is presented. For poppet valves, cracking pressure and pressure override curve are critical quality parameters. Fig. 7 shows the poppet geometry, inner detail and the initial mesh setup for the given case. There are overall seven zones in the mesh including two hexahedron and five tetrahedron zones. Five interfaces are built with two of them having sliding motion as shown in Fig. 8. UDF code is written using Newton's second law of motion to define the poppet motion by taking into account various forces on the moving poppet such as flow force, spring force, body mass. Flow force is an important factor which impacts the overall valve motion and hence needs accurate prediction in CFD simulation. Flow force methodology by Qinghui et al. [6] is leveraged for the same. At cracking pressure, the opening present between the poppet and the cavity is about 4-6 microns. Cracking can only be correctly modeled if mesh in this gap has sufficient elements.
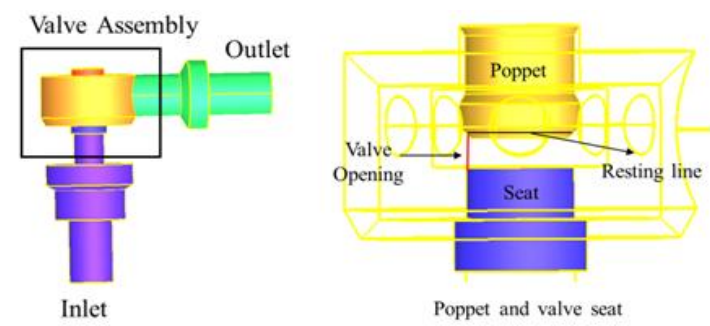

Fig. 7. Poppet geometry and valve assembly.

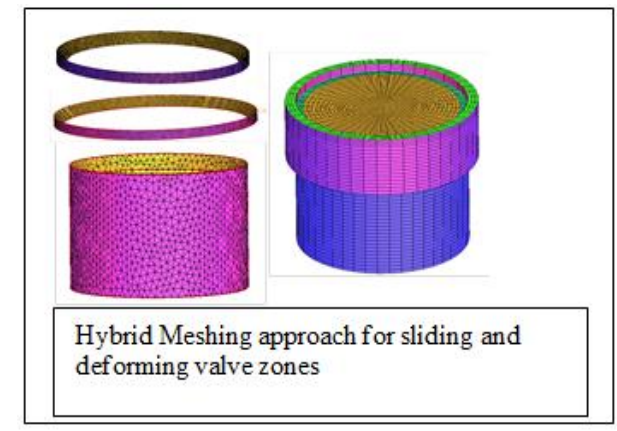

Fig. 8. Layering mesh for dynamic zone.

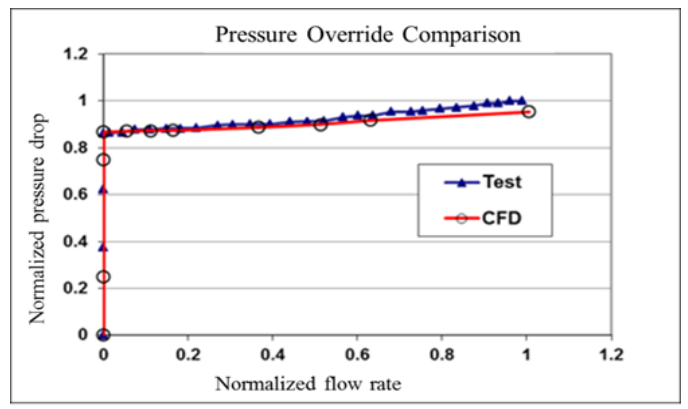

Fig. 9. Comparison of test and CFD results.

For pressure override prediction, the outlet is set at atmospheric pressure and inlet pressure is raised in steps to understand the flow behavior through the valve. A plot of normalized pressure against flow is generated which is known as the pressure override curve. Fig. 9 shows the comparison of CFD generated plot and the actual test plot. CFD results match well with test results with maximum error of $4 \%$. Using such kind of simulation, one can predictively design the control valve geometries to optimize the performance of a hydraulic circuit.

\section{CASE STUdy - Geroler MOTOR}

Third case study in this paper is the use of CFD simulation for performance prediction of hydraulic motor. Pressure drop is one of the important characteristics which directly affects mechanical efficiency of motor and fuel efficiency of a hydraulic system. In this paper, modeling approach of one such kind of Geroler motor for pressure drop prediction is presented. Typical construction of Geroler motor is shown in 
Fig. 10. The challenge in accurately predicting the pressure drop is to understand the motor dynamics and fluid cavity in different areas such as housing, valve plate orifices, Geroler pocket openings, etc. This decides selection of appropriate computational domain. Modeling variable fluid volume between star and rollers needs deforming zone technique which is complex and time consuming. Computational domain is appropriately selected at three different orientations in order to capture the least and maximum pressure drop without modeling Geroler pocket areas. Steady state simulation for three different orientations is performed and average pressure drop is reported. Fig. 11 shows comparison of CFD predictions and actual test observations. The results are in good agreement with test data with less than $5 \%$ error for all the flow rates. The validated methodology is being applied to study the effect of valve plate orifices, housing fluid cavities, Geroler pocket areas, etc. to optimize the design
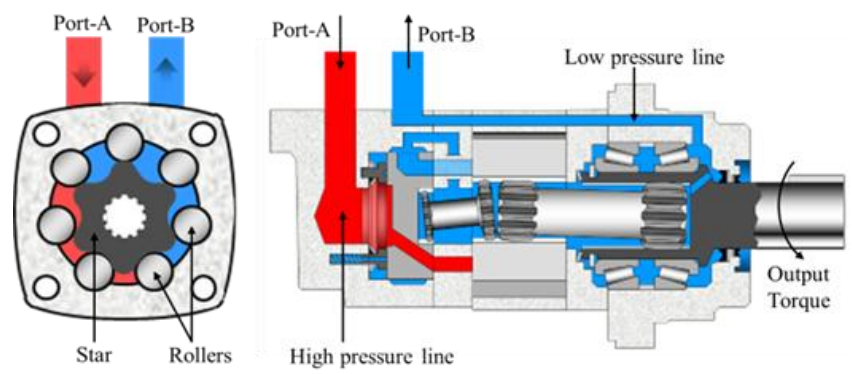

Fig. 10. Typical construction of Geroler motor.

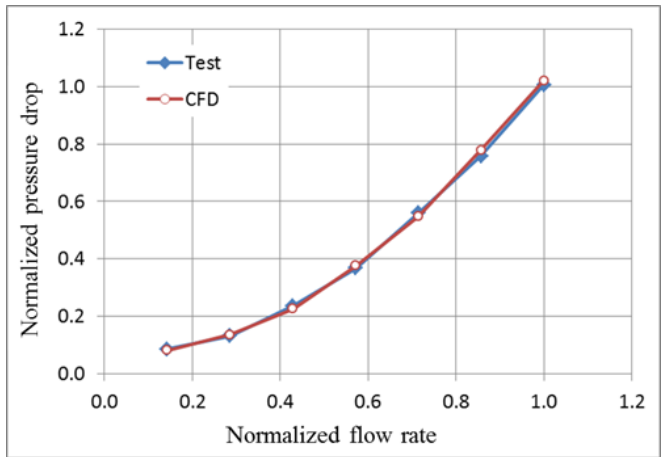

Fig. 11. Pressure drop - CFD vs. Test.

\section{SUMMARY}

Three case studies of CFD applications for hydraulic system design are described in this paper. In first case, CFD modeling approach for transient dynamic simulation for piston pump with multiphase physics is discussed to predict fill speed characteristics. Fill speed is one of the critical performance parameters for pump design qualification as it decides the maximum speed rating of the pump. CFD predictions are compared with test data for one of the pumps with less than $6 \%$ error. Similarly, the pressure override and pressure drop are important performance parameters for control valve and Geroler motor respectively. In second and third cases, simulation of control valve and Geroler motor is presented. CFD results for both the cases are in close agreement with test data with less than $5 \%$ error.

The validated CFD models are being applied for different designs to simulate the actual behavior of the fluid system, evaluate various design alternatives and develop optimized solution. CFD analysis not only saves the design and prototype building cost, but also more importantly accelerates the design cycle and improves speed to market. Thus, it has emerged as an integrated part of the design process.

\section{REFERENCES}

[1] S. Salutagi, M. Kulkarni, N. Gandhi, and R. Vale, "Numerical modeling to predict fill speed characteristics," presented at the NAFEMS World Congress, May 23-26, 2011, Boston, USA.

[2] ANSYS FLUENT 15.0 Theory Guide - Cavitation Models.

[3] N. Manring and Y. Zhang, "The improved volumetric efficiency of an axial piston pump utilizing a trapped volume design," Journal of Dynamic Systems, Measurement \& Control, vol. 123, pp. 479-487, September 2001

[4] A. Singhal, M. Athavale, H. Y. Li, and J. Yu, "Mathematical basis and validation of the full cavitation model," Journal of Fluids Engineering, vol. 124, pp. 617-624, September 2002.

[5] A. Kulkarni, M. Kulkarni, and S. Salutagi, "Numerical modelling of cavitation using energy approach," in Proc. the International Conference on Heat Transfer and Fluid Flow, August 11-12, 2014, Prague, Czech Republic.

[6] Y. Qinghui, S. Singh, A. Kulkarni, M. Kulkarni, P. Navale, and R. Navale, "Flow forces investigation through computational fluid dynamics and experimental study," in Proc. $9^{\text {th }}$ International Fluid Power Conf., March 24-26, 2014, AACHEN University, Germany.

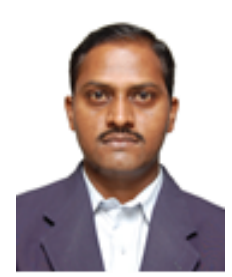

Shivayogi S. Salutagi received his B.E. degree in mechanical engineering from Shivaji University, Kolhapur, India in 2002. He completed his post-graduation in fluids \& thermal engineering from Walchand college of engineering, Sangli, Shivaji University, Kolhapur, India in 2005.

He joined Ashok Leyland, Chennai, India as a CFD engineer after his post-graduation and worked on CFD analysis projects for automotive applications till 2008 Then he joined Eaton India, Pune and currently he is working as the lead engineer (CFD) for hydraulics and aerospace products. His major areas of interest are cavitation, multiphase flow, conjugate heat transfer and dynamic mesh modeling.

Mr. Shivayogi Salutagi is a member of SAE. He has published several technical papers in multiple internationals conferences. He also guided 3 post-graduation student projects. 